\title{
ERROS DE DOSE RELACIONADOS A PROCEDIMENTOS DE ENFERMAGEM NA INFUSÃO ENDOVENOSA DE ANTIMICROBIANOS
}

\author{
Maria Lurdemiler Sabóia Mota ${ }^{1}$, Francisquinha Mota Vasconcelos², Luiz Eduardo Rodrigues Lins ${ }^{3}$, Ítalo Rigoberto
}

Cavalcante Andrade ${ }^{4}$

\begin{abstract}
RESUMO: A infusão de subdoses de antimicrobianos pode ser extremamente importante, pois interfere no desenvolvimento de resistência microbiana. O objetivo desse estudo foi analisar os erros de dose relacionados à infusão de antimicrobianos por via endovenosa. Trata-se de um estudo exploratório, realizado em uma unidade clínico-cirúrgica de um hospital público em Fortaleza-Ceará. Foram realizadas 174 observações de infusão de antimicrobianos com detecção de 59 erros ligados a dose menor do que a prescrita. Deste total (59) predominaram administrações de doses menores por resíduo nos dispositivos de infusão em 47,5\% (28). Os antimicrobianos mais frequentemente envolvidos nos erros foram: ceftriaxona 12 prescrições, ciprofloxacino 11 prescrições e vancomicina 8 prescriç̃oes. Os dados despertam para necessidade da ação conjunta da equipe de saúde na elaboração de rotinas para a infusão de antimicrobianos e para a educação continuada de técnicos e auxiliares na obediência a infusões completas das soluções para maior segurança terapêutica do paciente.

PALAVRAS-CHAVE: Erros de dose; Enfermagem; Antimicrobianos; Segurança do paciente.
\end{abstract}

\section{DOSE ERRORS RELATED WITH NURSING PROCEDURES IN INTRAVENOUS INFUSION OF ANTIMICROBIALS}

\begin{abstract}
The infusion of subdoses of antimicrobials may be extremely important, because it interferes in the microbial resistance development. The aim of this study was to analyze the errors related to dose infusion of intravenous antimicrobials. This is an exploratory study, conducted in a medical-surgical unit in a public hospital in Fortaleza- Ceará. 174 observations were made on antibiotics infusion with detection of 59 errors related to lower dose than the ones prescribed. Of this total (59) in 47,5\% (28) predominated administration of low doses related to the residue in infusion devices. The antimicrobial agents most frequently involved in errors were ceftriaxone (12 prescriptions), ciprofloxacin (11) and vancomycin (08 prescriptions). Data point out to the need of a joint action of the health team in the development of routines for antibiotics infusion and for the continuing education of technicians and assistants towards the obedience regarding complete infusion of solutions in order to increase the patient security regarding infusion therapy.
\end{abstract}

KEYWORDS: Dose errors; Nursing; Anti-bacterial agents; Patient safety.

\section{ERRORES DE DOSIS RELACIONADOS A PROCEDIMIENTOS EN ENFERMERÍA EN LA INFUSIÓN INTRAVENOSA DE ANTIMICROBIANOS}

RESUMEN: La infusión de subdosis de antimicrobianos puede ser extremamente importante, pues interfiere en el desarrollo de resistencia microbiana. El objetivo de este estudio fue analizar los errores de dosis relacionados con la infusión de antimicrobianos por vía intravenosa. Se trata de un estudio exploratorio, realizado en una unidad clínico-quirúrgica de un hospital público en Fortaleza- Ceará. Fueron realizadas 174 observaciones de infusión de antimicrobianos con detección de 59 errores relacionados a la dosis menor de la prescrita. Del total (59) predominaron administraciones de dosis menores por residuo en los dispositivos de infusión en 47,5\% (28). Los antimicrobianos más frecuentemente envueltos en los errores fueron: ceftriaxona 12 prescripciones, ciprofloxacina 11 prescripciones y vancomicina 08 prescripciones. Los datos despiertan la necesidad de acción conjunta del equipo de salud en la elaboración de rutinas para la infusión de los antimicrobianos y para la educación continuada de técnicos y auxiliares en la obediencia a infusiones completas de las soluciones para mayor seguridad terapéutica del paciente.

PALABRAS CLAVE: Errores de dosis; Enfermería; Antimicrobianos; Seguridad del paciente.

\footnotetext{
${ }^{1}$ Professora Assistente do Departamento de Enfermagem da Universidade de Fortaleza-UNIFOR. Doutora em Farmacologia pela Faculdade de Medicina da Universidade Federal do Ceará-FAMED-UFC.

${ }^{2}$ Graduando em Enfermagem da Universidade de Fortaleza-UNIFOR

${ }^{3}$ Graduando em Enfermagem da Universidade de Fortaleza-UNIFOR.

${ }^{4}$ Enfermeiro Especialista em Terapia Intensiva. Professor auxiliar da UNIFOR.

Autor correspondente:

Maria Lurdemiler Sabóia Mota

Universidade de Fortaleza

Rua Israel Bezerra, 1080 - 60135-460 - Fortaleza-CE, Brasil

Recebido: 02/04/09

E-mail:mila269@terra.com.br

Aprovado: 08/09/09
} 


\section{INTRODUÇÃO}

Antimicrobianos são definidos como substâncias que provocam morte ou inibição do crescimento de microorganismos. Podem ser produzidos por bactérias ou por fungos ou podem ser total ou parcialmente sintetizados. De todos os fármacos, estão entre os mais comumente prescritos e também entre os mais utilizados de modo incorreto ${ }^{(1)}$.

A via endovenosa é recomendada para os antimicrobianos diante de infecções graves, nas quais há a necessidade de rápidas e mantidas concentrações do fármaco. Para ser eficaz contra um microorganismo causador de um processo infeccioso, o antimicrobiano ativo deve alcançar, no foco de infecção, concentração suficiente para matar ou inibir o agente patogênico ${ }^{(1)}$.

O conceito farmacológico de dose, diz da quantidade de medicamento que um paciente deve receber para modificar seu estado de enfermidade. Sendo, em termos farmacocinéticos, o objetivo das doses horárias manter o nível plasmático do medicamento dentro do limite mínimo necessário para a presença do terapeuticamente esperado e máximo de distribuição plasmática, em que a presença de eventos adversos supera o tolerável pelo paciente ${ }^{(2)}$.

A relação entre a dose administrada e a concentração de fármaco presente na circulação sistêmica, bem como no local de ação, depende das múltiplas variáveis comuns a todo o processo farmacodinâmico e farmacocinético: absorção, distribuição, metabolismo, ligação a proteínas, ações em receptores e eliminação. Qualquer passo deste processo está sujeito a diferenças interindividuais e intraindividuais $^{(2)}$. As ações de enfermagem na implementação terapêutica podem modificar todo esse processo, visto ser de responsabilidade deste profissional a administração do medicamento. Dentro deste contexto, o ato e dever da aferição de dose ganha extrema relevância.

Quando se administra um fármaco por via endovenosa, toda a dose penetra na circulação sistêmica, por conseguinte, esta via possui disponibilidade sistêmica de 100\%. Em termos farmacológicos, as doses precisam ser rigorosamente obdecidas para que se consiga perfis de disponibilidade sistêmica ideais para cada fármaco prescrito e administrado $^{(3)}$.

Doses maiores e também volumes maiores de diluentes podem igualmente influenciar a forma de preparo e administração pelo pessoal de enfermagem e isto pode alterar os perfis farmacocinéticos e, consequentemente, farmacodinâmicos de cada fármaco ${ }^{(4)}$. A vancomicina (glicopeptídeo de amplo espectro de ação antimicrobiana), por exemplo, em dose de 500 mg deve ser diluída em $100 \mathrm{ml}$ e infundir em 60 minutos $^{(4)}$. Entretanto, se for diluída em menor volume, expõe o paciente à possibilidade de reações adversas, o que poderia levar à interrupção de tratamento com troca de terapêutica. Adicionalmente, se a equipe de enfermagem deixar resto de solução no equipo de infusão, a perda é proporcionalmente maior quanto mais concentrada for a solução e isso pode ser considerado erro de dose $\mathrm{e}^{(5-6)}$.

A administração da dose, concentração e tempo de infusão corretos de um medicamento, de forma geral, dependem, em grande parte da equipe de enfermagem. Concentrações maiores (erros de dose) e infusões demasiado rápidas podem levar desde reações locais, com inflamação, infecção e necessidade de tratamento, até reações cutâneas e sistêmicas gerando equívocos que levam à mudança desnecessária do tratamento prescrito ${ }^{(7)}$.

Para fazer o medicamento prescrito chegar à corrente sanguínea dispõe-se de diversas marcas de equipos, extensores para equipos e dispositivos para acesso a veia (cateteres longos, curtos e de breve e longa permanência). Dependendo do fabricante, temos todos estes dispositivos com capacidades diferentes de preenchimento. Assim, existem equipos com capacidade de preenchimento de: $8 \mathrm{ml}$, $5 \mathrm{ml}$ e $20 \mathrm{ml}$; extensores com capacidade semelhantes e cateteres com capacidade variando de $1 \mathrm{ml}$ (“scalp”) a $2 \mathrm{ml}$ ("intracath"). Considera-se a dose de medicamento completamente infundida quando não restar solução medicamentosa nestes dispositivos. Trabalho brasileiro norteador de práticas na área ${ }^{(4)}$ recomenda a adição de $20 \mathrm{ml}$ de soro fisiológico ou solução compatível com o antimicrobiano ao final da dose intravenosa na bolsa do medicamento, deixando o restante da dose fluir.

A interferência das ações da enfermagem nos perfis farmacocinéticos e nas curvas farmacodinâmicas pode ser deduzida pela presença de resposta no paciente. Isto ocorre por ser a administração da dose, concentração e tempo de infusão corretos de um antibiótico, de forma geral, dependente, em grande parte das ações da equipe de enfermagem ${ }^{(5)}$.

Desta forma, o presente estudo é relevante à medida que vislumbra o desenvolvimento de uma assistência de enfermagem que traga a perspectiva de melhora de parâmetros clínicos, através da obediência 
a princípios fundamentais de prática para segurança farmacológica do paciente. Portanto, através da observação e cumprimento de regras práticas no preparo e administração de antimicrobianos em que se busque o princípio básico da infusão de doses corretas, o paciente pode ser clinicamente beneficiado pela manutenção de concentrações plasmáticas constantes e, com isso, probabilidade melhor e maior de resposta terapêutica mais efetiva e rápida.

Assim, neste estudo tem-se o objetivo: analisar os erros de dose relacionados a procedimentos de enfermagem na administração de antimicrobianos por via endovenosa.

\section{MÉTODO}

Trata-se de um estudo com delineamento exploratório, que visa obter informações detalhadas da variável erro de dose relacionada à técnica de infusão endovenosa de antimicrobianos em uma unidade de internamento clínico cirúrgico, buscando determinar a frequência e as possíveis causas associadas à ocorrência do evento. Considerou-se como erro de dose a administração ao paciente de uma quantidade maior ou menor que a prescrita ou administração de dose duplicada. Isto é, uma ou mais doses administradas além da prescrita.

A população em estudo foi composta pelas situações em que houve detecção de erro de medicação ligada à dose administrada de antimicrobianos, ou seja, 59 erros identificados em um total de 174 observações. A unidade de análise foi a administração de antimicrobiano.

O estudo foi conduzido em uma instituição hospitalar pública de atendimento especializado e de ensino do município de Fortaleza-Ceará, referenciado como membro da rede sentinela da Agência Nacional de Vigilância Sanitária-ANVISA. Esse local constitui centro de excelência na assistência à saúde. Atende vários graus de complexidade nos níveis assistenciais em unidades ambulatoriais, de procedimentos especializados, internação, urgência, prevenção, tratamento e reabilitação, de natureza clínica e/ou cirúrgica, além de serviços complementares de diagnóstico e tratamento em diversas especialidades médicas. É entidade autárquica, integrada ao Sistema Único de Saúde-SUS e possui como finalidade, o ensino, a pesquisa e a assistência médico-hospitalar. A unidade de clínica médica, ligada ao setor de emergência, foi selecionada por possuir leitos destinados a pacientes portadores de doenças crônico-degenerativas e em pré e pós-operatório que usualmente utilizam antimicrobianos por um longo período, englobando diversos grupos farmacológicos.

A investigação foi realizada após a aprovação do Comitê de Ética em Pesquisa da instituição e registrada com o n. de protocolo: 131101/08. Todos os participantes foram orientados sobre o estudo, aceitaram, por escrito, serem observados e assinaram o termo de consentimento livre e esclarecido, conforme a Resolução n. ${ }^{0}$ 196/96 do Conselho Nacional de Saúde ${ }^{(8)}$.

Para a coleta dos dados foram realizadas observações não-participantes e diretas das atividades dos profissionais de enfermagem responsáveis pelo preparo e administração de medicamentos seguindo um roteiro previamente estruturado.

Como possíveis causas para erro de dose, considerou-se os equipos e extensores retirados do paciente preenchidos ou parcialmente preenchidos e restos de solução nas bolsas de soro. Concluída a infusão, os pesquisadores solicitavam que o responsável pela administração do antimicrobiano não colocasse no lixo os dispositivos utilizados para a infusão. Após receber os dispositivos utilizados o pesquisador colocava todo resto de solução em um copo descartável e com uma seringa graduada de $1 \mathrm{a} 20 \mathrm{ml}$, media os restos de solução de equipos e bolsas de infusão retirados do paciente a cada horário. As perdas por horário foram anotadas e somadas ao final de 24 horas.

Após as observações da administração dos antimicrobianos, os observadores confrontaram as informações obtidas com as prescrições, a fim de detectar se ocorreu algum erro de leitura da dose administrada. Durante o preparo foi observada apenas a quantidade e compatibilidade da solução diluente utilizada.

O conceito de erro de dose adotado foi o descrito pela American Society of Health System Pharmacists ${ }^{(9)}$. A classificação farmacológica dos antimicrobianos identificados nos erros foi realizada segundo o sistema anatômico terapêutico químicoATC do WHO Collaborating Center for Drug Statistics Methodology ${ }^{(10)}$, e empregado pelo Centro Colaborador para o Monitoramento Internacional de Medicamentos.

Os dados coletados foram transferidos para um banco de dados. A análise estatística foi realizada empregando o software SPSS 11.5 e consistiu de análise descritiva univariada com determinação das frequências absolutas e relativas. 


\section{RESULTADOS E DISCUSSÃO}

No período de setembro a outubro de 2008 foram realizadas 174 observações da administração de antimicrobianos, nas quais foram identificados 59 (33,9\%) erros de dose, classificados conforme Tabela 1.

Tabela 1 - Distribuição dos erros de dose estratificados conforme a causa. Fortaleza, 2008

\begin{tabular}{lcc}
\hline Causa de erros de dose & N. & $\%$ \\
\hline $\begin{array}{l}\text { Dose menor por equipo e extensores } \\
\text { preenchidos ao final da infusão }\end{array}$ & 28 & 47,5 \\
$\begin{array}{l}\text { Dose maior ou menor por volume de } \\
\text { solução diluente inadequado }\end{array}$ & 14 & 23,7 \\
$\begin{array}{l}\text { Dose menor por volume de diluição } \\
\text { inadequado e equipo e extensores } \\
\text { preenchidos ao final da infusão }\end{array}$ & 17 & 28,8 \\
\hline Total de observações & 59 & 100 \\
\hline
\end{tabular}

A dose menor do que a prescrita foi identificada pelo resíduo presente no equipo e/ou bolsa de soro ao final da infusão, enquanto doses maiores foram consideradas em relação a erros de diluição com administração de concentrações maiores do que as prescritas. Do total de observações com erros houve predominância absoluta da administração de doses menores do que as constantes na prescrição médica 28 (47,5\%). Este fato, apesar de não corroborado pela literatura, que é rica em relatos de administração de doses maiores, omissão de doses ou doses duplicadas $^{(11)}$, possivelmente pode estar relacionado ao fato da preocupação primária deste estudo ter sido observar o final do procedimento de administração de antimicrobianos com o acompanhamento do descarte do material utilizado.

Independente da significância estatística deste dado, é importante considerar que a infusão de doses inferiores às prescritas por repetidas vezes levará, com certeza, ao insucesso da terapêutica o que coloca a segurança do paciente em risco. O que preocupa é que, quase sempre, a falha terapêutica é atribuída ao medicamento não sendo vislumbrada a possibilidade de falha no preparo e administração do antimicrobiano. Assim, este dado parece apontar para necessidade urgente de estudos mais completos e aprofundados nesta área.

Estudo semelhante realizado em Porto Alegre ${ }^{(6)}$ demonstrou que em $9 \%$ das observações realizadas para infusão de um antimicrobiano específico o equipo não foi lavado ou foi retirado cheio. Em comparação, os nossos dados são corroborados por esse achado partindo-se do pressuposto que na unidade de internamento por nós estudada não há rotina de lavagem dos equipos como no serviço avaliado pelas autoras onde se recomenda, por rotina, a lavagem dos dispositivos utilizados no processo de infusão com 20 $\mathrm{ml}$ de solução(4).

Na Tabela 2 estão dispostos o montante de perdas para doses planejadas e prescritas para utilização em 24 horas, considerando-se os dez antimicrobianos mais encontrados nas prescrições avaliadas. Avaliamos o montante de perda para este período exclusivo (24 horas). Portanto, não consideramos as perdas acumuladas para todo o tratamento.

A utilização de volumes menores ou maiores do que os recomendados pela literatura pode alterar as concentrações do antimicrobiano em solução e, por consequência, alterar perfis farmacocinéticos de distribuição e farmacodinâmicos em relação a receptores e ação.

Com base em estudos existentes sobre estas variáveis se reconhece que muitas questões ainda permanecem sem resposta em função das definições de alteração de resposta relacionada a diluições variarem de acordo com diferentes autores. Assim como a constatada existência de fatores de confusão nas pesquisas e outros problemas metodológicos devido à dificuldade de controlar algumas variáveis farmacocinéticas e farmacodinâmicas ${ }^{(7,9-10)}$.

Dado preocupante é exposto na Tabela 2, em que vários antimicrobianos foram administrados em doses menores do que as prescritas devido a erro básico de infusão. Conforme a literatura ${ }^{(12-13)}$ os efeitos da redução de dose variam com o grupo farmacológico e com condições orgânicas individuais como função hepática e renal do paciente. Entretanto, tais dados nos chamam a atenção para a necessidade urgente de revisão nos processos gerais de enfermagem em relação à administração de medicamentos.

Para padronização de doses e de intervalos de administração de antimicrobianos são utilizados parâmetros farmacodinâmicos capazes de quantificar a atividade antimicrobiana para determinado patógeno, e parâmetros farmacocinéticos capazes de permitir inferências sobre correlações ligadas à distribuição e eliminação dos antimicrobianos que se traduzirão na área sob a curva. Assim, as concentrações inibitórias mínima e bactericida mínima são utilizadas como valores críticos para interpretação da relação entre os 
parâmetros farmacocinéticos que determinam a eficácia do antimicrobiano e a sua potência ${ }^{(14)}$.

Tabela 2 - Distribuição dos dez antimicrobianos com média de perdas diárias identificadas no processo de administração. Fortaleza, 2008

\begin{tabular}{lccc}
\hline Antimicrobianos por número de prescrições & Dose prescrita/24h & Perdas/24h & Dose administrada \\
\hline Ceftriaxona - 12 Prescrições & $2000 \mathrm{mg} / 24 \mathrm{~h}$ & $13 \mathrm{ml} / 130 \mathrm{mg}$ & $1870 \mathrm{mg}$ \\
Cefepime - 3 Prescrições & $2000 \mathrm{mg} / 24 \mathrm{~h}$ & $18 \mathrm{ml} / 180 \mathrm{mg}$ & $1820 \mathrm{mg}$ \\
Ciprofloxacino - 11 Prescrições & $400 \mathrm{mg} / 24 \mathrm{~h}$ & $14 \mathrm{ml} / 28 \mathrm{mg}$ & $372 \mathrm{mg}$ \\
Clindamicina - 4 Prescrições & $2700 \mathrm{mg} / 24 \mathrm{~h}$ & $24 \mathrm{ml} / 216 \mathrm{mg}$ & $2484 \mathrm{mg}$ \\
Oxacilina - 6 Prescrições & $2000 \mathrm{mg} / 24 \mathrm{~h}$ & $30 \mathrm{ml} / 300 \mathrm{mg}$ & $1700 \mathrm{mg}$ \\
Metronidazol - 7 Prescrições & $1500 \mathrm{mg} / 24 \mathrm{~h}$ & $12 \mathrm{ml} / 60 \mathrm{mg}$ & $1460 \mathrm{mg}$ \\
Piperacilina e tazobactama sódica - 2 Prescrições & $5000 \mathrm{mg} / 24 \mathrm{~h}$ & $16 \mathrm{ml} / 133 \mathrm{mg}$ & $4866 \mathrm{mg}$ \\
Penicilina cristalina - 4 Prescrições & $10 \mathrm{milhões} / 24 \mathrm{~h}$ & $28 \mathrm{ml} / 0,93 \mathrm{milhões}$ & $9,1 \mathrm{milhões}$ \\
Sulbactam e ampicilina - 2 Prescrições & $3000 \mathrm{mg} / 24 \mathrm{~h}$ & $5 \mathrm{ml} / 150 \mathrm{mg}$ & $2850 \mathrm{mg}$ \\
Vancomicina - 8 Prescrições & $2000 \mathrm{mg} / 24 \mathrm{~h}$ & $10 \mathrm{ml} / 50 \mathrm{mg}$ & $1950 \mathrm{mg}$ \\
\hline
\end{tabular}

Total de Prescrições - 59

A interferência dos erros de dose de antimicrobianos sobre os componentes farmacocinético e farmacodinâmico e a interrelação com a resistência microbiana já foi pontuada na literatura ${ }^{(14)}$. As autoras chamam a atenção para o fato de que, independentemente do número de vezes em que doses incompletas de antimicrobianos são administradas, o que deve ser considerado é o fato de que este erro de administração pode levar a concentrações plasmáticas inferiores à concentração inibitória mínima, exercendo pressão seletiva sobre o microrganismo. A pressão seletiva é um dos fatores de risco para o aparecimento da resistência microbiana ${ }^{(14)}$.

Para a segurança do paciente é preceito farmacológico básico que a dose prescrita seja totalmente infundida a fim de que se possa prover o cliente da possibilidade de resposta terapêutica adequada. De acordo com as leis vigentes no Brasil é de responsabilidade do enfermeiro a administração de medicamentos $^{(11-12)}$. Compreendendo-se como administração do medicamento o processo final de chegada da substância terapêutica ao organismo do paciente, estando envolvida neste processo a obrigatoriedade de obediência à prescrição médica com tudo que a envolve: dose, forma farmacêutica e via( ${ }^{(2)}$.

Entretanto, é importante salientar que na visão mais ampla da compreensão global dos erros com medicamentos as administrações de doses completas são de responsabilidade de todos os envolvidos no processo, desde o preparo até a infusão. Utilizar equipamentos que evitem doses incompletas pode ser uma das alternativas para minimizar o problema ${ }^{(13)}$. Todavia, nossos resultados chamam a atenção para o fato de que mesmo na utilização deste tipo de equipamento (bombas infusoras) o treinamento da equipe de enfermagem tem que existir no sentido de que toda a solução contendo o medicamento atinja a corrente sanguínea.

A Tabela 3 traz os três antimicrobianos, cujas administrações foram observadas por um maior número de vezes. Tal fato ocorreu por serem estes os mais frequentemente escolhidos pelos médicos assistentes na unidade onde se realizou o estudo. Os dados são preocupantes quando correlacionamos as doses sub-clínicas administradas com os grupos aos quais os três fármacos pertencem e sua importância no controle clínico de diversas infecções.

A ceftriaxona foi o antimicrobiano mais envolvido com o erro de infusão. É classificada como uma cefalosporina de terceira geração com amplo espectro de ação e indicada no tratamento de infecções do trato biliar, ósseas, do sistema nervoso central, do trato geniturinário, gonorreia, pneumonia, septicemia bacteriana, infecções de pele e tecidos moles ${ }^{(2)}$. Sua ação bactericida depende de sua capacidade em alcançar e unir-se às proteínas que ligam penicilina, localizadas nas membranas citoplasmáticas bacterianas $^{(3)}$. 
Tabela 3 - Antimicrobianos com maior número de prescrições e envolvidos com erros de dose durante o processo de administração. Fortaleza, 2008

\begin{tabular}{lccc}
\hline Antimicrobianos por número de prescrições & Dose prescrita/24h & Perdas/24h & Dose administrada \\
\hline Ceftriaxona - 12 Prescrições & $2000 \mathrm{mg} / 24 \mathrm{~h}$ & $13 \mathrm{ml} / 130 \mathrm{mg}$ & $1870 \mathrm{mg}$ \\
Ciprofloxacino - 11 Prescrições & $400 \mathrm{mg} / 24 \mathrm{~h}$ & $14 \mathrm{ml} / 28 \mathrm{mg}$ & $372 \mathrm{mg}$ \\
Vancomicina - 8 Prescrições & $2000 \mathrm{mg} / 24 \mathrm{~h}$ & $10 \mathrm{ml} / 50 \mathrm{mg}$ & $1950 \mathrm{mg}$ \\
\hline Total de Prescrições -59 & - & - & - \\
\hline
\end{tabular}

O risco que envolve as subdoses de ceftriaxona reside na possibilidade de seleção de bactérias produtoras de beta-lactamases, que são enzimas que provocam a hidrólise de componente do anel betalactâmico, inativando o antimicrobiano. Estas enzimas são produzidas tanto por bactérias Gram-positivas como por Gram-negativas ${ }^{(3)}$.

A ciprofloxacina também apareceu muito nas prescrições analisadas. A importância clínica deste antimicrobiano, uma quinolona de segunda geração, está na sua utilização em diversas situações infecciosas. O grande risco na utilização deste fármaco, principalmente quando de sua utilização em infecções do trato respiratório, é a ocorrência de resistência por parte de pneumococos, clamidias e micoplasmas $^{(3)}$.

A Tabela 3 também chama a atenção para as doses menores infundidas durante a administração de vancomicina, glicopeptídeo de intervalo terapêutico estreito. A grande preocupação com a infusão de doses menores de vancomicina é com a resistência dos microrganismos. A redução da sensibilidade do Staphylococcus aureus à vancomicina representa uma grande preocupação, por se tratar de uma das últimas opções terapêuticas a esse microrganismo. Outra questão alvo de maior ou igual preocupação é a resistência de Enterococcus à vancomicina ${ }^{(14)}$.

Reconhecidamente, o mau emprego dos antimicrobianos na terapêutica e na profilaxia de infecções constitui uma das principais causas do aumento da resistência bacteriana. A este sério problema, devese acrescentar a ineficácia terapêutica, o risco de efeitos adversos e o custo que estes problemas representam para a economia dos pacientes ou do Estado. Desta forma, o uso clínico dos antimicrobianos exige o conhecimento de critérios e princípios gerais, que permitam o seu emprego racional e a obtenção dos resultados satisfatórios desejados ${ }^{(12)}$.

Neste contexto, fica evidente que a infusão de doses menores pode comprometer a resposta terapêutica do antimicrobiano. Este tipo de erro pode impedir que o antimicrobiano alcance o local da infecção, mantenha concentrações suficientes no foco da infecção para exercer sua ação e permaneça no local tempo suficiente para inibir a multiplicação ou matar os micro-organismos.

\section{CONSIDERAÇÕES FINAIS}

Os fatores cruciais a considerar na utilização de antimicrobianos consistem na concentração inibitória mínima e na concentração bacteriana mínima do fármaco contra determinado microorganismo. Desta forma, a dose deve ser planejada e administrada de modo a produzir concentrações bactericidas ou bacteriostáticas no local da infecção.

O maior problema encontrado por este estudo foi a administração de doses menores do que as prescritas por infusão incompleta da solução constante em bolsas de soro, equipos, extensores e dispositivos utilizados para o acesso venoso. Nossos resultados apontam para a necessidade urgente de modificações nas ações de enfermagem no que se refere a este ponto e, principalmente, quando se tratar da infusão endovenosa de antimicrobianos.

Vários grupos de antimicrobianos estiveram envolvidos nos erros de infusão, sendo os mais frequentes ceftriaxona, uma cefalosporina de inquestionável importância clínica, ciprofloxacino, uma quinolona de primeira escolha em vários processos infecciosos e vancomicina, um glicopeptídeo apresentado como uma das últimas opções terapêuticas nas infecções por Staphylococcus aureus. Este dado é extremamente preocupante pelo grande risco das doses menores levarem a concentrações teciduais insuficientes do fármaco e a seleção de microorganismos resistentes.

Os dados apresentados apontam para a importância do estímulo a ação conjunta de médicos, farmacêuticos e enfermeiros para a elaboração de 
rotinas para a infusão adequada de antimicrobianos e para o despertar pela educação continuada de técnicos e auxiliares de enfermagem, no sentido de que a obediência as regras básicas de cuidado, como a infusão completa das soluções medicamentosas, podem contribuir para a segurança do paciente.

\section{REFERÊNCIAS}

1. Tavares W, Magalhães GAP, Sangenis LHC, Goulart MEM, Reis VLL. Antibióticos e quimioterápicos para o clínico. São Paulo: Atheneu; 2007.

2. Grahame-Smith DG, Aronson JK. Interações medicamentosas. In: Grahame-Smith DG, Aronson JK. Tratado de farmacologia clínica e farmacoterapia. Rio de Janeiro: Guanabara Koogan 2004. p.98-109.

3. Labarca JL. Nuevos conceptos en farmacodinamia, debemos repensar cómo administramos antimicrobianos? Rev Chilena Infectol. 2002;19(suppl 1):33-7.

4. Hoefel HHK, Lautert L. Administração endovenosa de antibióticos e resistência bacteriana: responsabilidade da enfermagem. Rev Eletron Enferm. 2006 [acesso em 2009 Mar 01]8(3):441-9. Disponível: http://www.fen.ufg. br/revista/revista8_3/v8n3a15.htm

5. Hoefel H, Lautert L. Errors committed by nursing technicians and assistants in administrating antibiotics. Am J Infec Control. 2006;34:437-42.

6. Hoefel, H. Vancomycin administration in an universitary hospital at general surgical units inpatients. Online OBJN [periódico da Internet]. 20043 (1) [acesso 2008 Out 15] Disponível: http://www.uff.br/nepae/ objn301hoefeletal.htm

7. American Association of Hospital Pharmacists. ASHP. Guideline on preventing medication errors in hospitals. Am J Hosp Pharm. 1993;50:305-14.

8. Ministério da Saúde (BR). Conselho Nacional de Saúde. Resolução n.196, de 10 de outubro de 1996. Aprova as Diretrizes e Normas Regulamentadoras de Pesquisas com seres humanos. [acesso em 2008 Mar 30] Disponível: http://conselho.saude.gov.br/docs/ Resolucoes/Reso196.doc

9. American Society of Health-System Pharmacists. ASHP. technical assistance bulletin on hospital drug distribution and control. Am J Health-System Pharmacy [online] 1980. [Acesso em 2008 Out 5]. Disponível: http:/ /www.ajhp.org/cgi/external_ref?access_num=7405941\& displayid=147468\&link_type=INFOTRIEVE .
10. World Health Organization Collaborating Centre For Drug Statistics Methodology. Anatomical Therapeutic Chemical (ATC) index with Defined Daily Doses (DDDs) [acesso em 2008 Out 10]. Disponível: http:// www.whocc.no/atcddd.

11. Marques TC, Reis AMM, Silva AEBC, Gimenes FRE, Opitz SP. Erros de administração de antimicrobianos identificados em estudo multicêntrico brasileiro. Rev Bras Cienc Farm. 2008 Abr/Jun;44(2):380-1.

12. Ford NA, Drott HD, Cieplinski-Robertson. Administrations of IV medications via soluset. Ped Nursing. 2003;29(4):283-6.

13. Yu WL. Cefepime MIC as a predictor of the extendedspectrum beta-lactamase type in Klebsiela pneumoniae, Taiwan. Emer Infec Diseas. 2002;8(5):522-4.

14. Muto C, Jernigan JA, Ostrowsky BR, Richet HE, Jarvis WR, Boyce JM, et al. SHEA guideline for preventing nosocomial transmission of multidrug-resistant strains of Sthaphylococcus aureus and Enterococcus. Infect Control Hos Epidemiol. 2003;24:362-86. 